\title{
Efficacy of Dose-Escalated Radiotherapy for Recurrent Colorectal Cancer
}

\author{
Sunmi Jo, Yunseon Choi ${ }^{1}$, Sung-Kwang Park ${ }^{1}$, Jin-Young Kim, Hyun Jung Kim, Yun-Han Lee ${ }^{2}$, \\ Won Yong Oh, Heunglae Cho ${ }^{1}$, Ki Jung Ahn ${ }^{1}$ \\ Department of Radiation Oncology, Inje University Haeundae Paik Hospital, Inje University School of Medicine, Busan, ${ }^{1}$ Department of \\ Radiation Oncology, Inje University Busan Paik Hospital, Inje University School of Medicine, Busan, ${ }^{2}$ Department of Radiation Oncology, \\ Yonsei University Health System, Yonsei University College of Medicine, Seoul, Korea
}

Purpose: This study aimed to evaluate the effects of radiotherapy (RT) on progression-free survival (PFS) for patients with recurrent colorectal cancer.

Methods: We reviewed the records of 22 patients with recurrent colorectal cancer treated with RT between 2008 and 2014. The median radiation dose for recurrent disease was 57.6 Gy (range, 45-75.6 Gy). Patients were divided into 2 groups according to the type of RT: patients underwent RT without previous history of irradiation $(n=14)$ and those treated with secondary RT (reirradiation: $\mathrm{n}=8$ ) at the time of recurrence.

Results: The median follow-up period was 24.9 months (range, 4.5-66.6 months). Progression was observed in 14 patients (including 8 with loco-regional failure and 9 with distant metastases). Distant metastases were related to the RT dose $(<70$ $\mathrm{Gy}, \mathrm{P}=0.031$ ). The 2-year loco-regional control (LRC), PFS, and overall survival $(\mathrm{OS})$ rates were $74.6 \%, 45.1 \%$, and $82.0 \%$, respectively. The LRC rate was not different between the patients treated with RT for the first time and those treated with reirradiation $(\mathrm{P}=0.101,2$-year LRC $79.5 \%$ vs. $41.7 \%)$. However, reirradiation was related to poor PFS $(\mathrm{P}=$ $0.022)$ and $\mathrm{OS}(\mathrm{P}=0.002)$. An escalated RT dose $(\geq 70 \mathrm{~Gy})$ was associated with a higher PFS $(\mathrm{P}=0.014,2$-year PFS $63.5 \%$ vs. $20.8 \%)$.

Conclusion: Salvage RT for locally recurrent colorectal cancer can be offered when surgery is impossible. Dose-escalated RT shows a possible benefit in reducing the risk of progression.

Keywords: Disease-free survival; Recurrence; Rectal neoplasms; Radiotherapy; Dose escalation

\section{INTRODUCTION}

Most patients with colorectal cancer without distant metastases are treated with surgical excision at initial diagnosis. However, radiotherapy (RT) still plays an important role, in combination with surgery and chemotherapy, in multimodal treatment for patients with rectal cancer. In particular, according to the current National

Received: October 8,2015 • Accepted: December 23, 2015

Correspondence to: Yunseon Choi, M.D.

Department of Radiation Oncology, Inje University Busan Paik Hospital, Inje University School of Medicine, 75 Bokjiro, Busanjin-gu, Busan 47392, Korea Tel: +82-51-890-8606, Fax: +82-51-891-1754, e-mail: rtyoon@gmail.com

(c) 2016 The Korean Society of Coloproctology

This is an open-access article distributed under the terms of the Creative Commons Attribution NonCommercial License (http://creativecommons.org/licenses/by-nc/4.0) which permits unrestricted noncommercial use, distribution, and reproduction in any medium, provided the original work is properly cited.
Comprehensive Cancer Network (NCCN) guidelines, for patients with advanced stage rectal cancer (T3-4 or N1-2), neoadjuvant chemoradiotherapy before surgery is recommended to achieve the best results [1].

Performing a total-mesorectal excision (TME) significantly was reported to reduce the local recurrence rate in patients with rectal cancer [2]. According to studies that were completed before the TME was commonly used for rectal-cancer treatment [3-6], up to $33 \%$ of patients experienced local recurrence, even after curative surgery. However, local recurrence occurs in $4 \%-10 \%$ of patients in the era of the TME $[2,7]$. As a result, the role of RT tends to be diminishing compared to that in the pre-TME era.

Surgical treatment is considered a mainstay of treatment for recurrent rectal cancer [5]. According to a report by Palmer et al. [8], the 5-year survival rate was $57 \%$ in patients treated with a curative resection. In addition, Bouchard and Efron [7] reported 
that patients with recurrence who were successfully managed with combined surgery and chemoradiotherapy showed better survival, with a 5-year OS rate of up to $35 \%$, compared to those who were treated without surgical treatment. For patients who did not receive surgery for their recurrent rectal cancer, the reported 5-year overall survival (OS) rate was as low as $<5 \%$ when treated with supportive care or palliative treatment [9]. However, only half of all patients are reported to be able to be treated with reoperation owing to tumor extension into or fixation to other pelvic structures [10]. Moreover, an R0 resection can only be achieved in less than one-third of the patients treated with reoperation [10].

In this study, we aimed to evaluate the efficacy of salvage RT in treating patients with loco-regionally recurrent colorectal cancer. As data about RT for the treatment of recurrent colorectal cancer are still lacking [11], this study could provide new information in this field. The primary endpoint of this study was the progressionfree survival (PFS) after salvage RT for the treatment of recurrent disease. In addition, we sought to determine the prognostic factors related to treatment failure after a salvage RT for the treatment of recurrent colorectal cancer. We also tried to determine the effect of radiation dose escalation on the RT outcomes.

\section{METHODS}

\section{Patients}

We retrospectively evaluated 22 patients who were clinically diagnosed with loco-regional relapses of colorectal cancer (confirmed using radiology and/or pathology) without distant metastases. All 22 patients with recurrent colorectal cancer were treated with salvage RT without surgery between June 2008 and to October 2014 at Busan Paik Hospital and Haeundae Paik Hospital. At the time of the diagnosis of recurrence, all patients were evaluated with computed tomography (CT); among them, 20 (90.9\%) received positron emission tomography (PET) to confirm recurrence. Pathological confirmation (biopsy) was performed in the other 2 patients who experienced relapses. This study was approved by the institutional review board of Inje University Busan Paik Hospital.

\section{Treatment}

None of the patients received any surgical resection for recurrence. Chemotherapy was performed according to the physician's preference. Combined chemotherapy with 5-fluorouracil, capecitabine, tegafur, oxaliplatin, and doxifluridine was used simultaneously with RT for salvage treatment. Sequential chemotherapy with the FOLFOX (Folinic acid, Fluorouracil, and Oxaliplatin) regimen, irinotecan, and capecitabine was administered after RT. For RT, all patients underwent CT simulation. All patients received 3-dimensional conformal RT, except for 2 patients who were treated with intensity-modulated radiotherapy (IMRT) for reirradiation. For RT, a 6- to 10-MV energy was used. Irradiation was performed with a daily dose of $1.8-2$ Gy with a margin of $1-3 \mathrm{~cm}$ from the beam to the tumor. The margin of the reduced field was defined as $0.3-0.8 \mathrm{~cm}$ of the gross tumor. The decision to use radiation dose escalation was made depending on physician's preference and the distance of the tumor from critical organs including the vagina, bladder, and anus, among others. The history of previous RT also influenced the decision making for dose-escalated RT.

\section{Follow-up}

Follow-up imaging using CT or PET was performed from 2 to 4 times in the year after treatment. Loco-regional failure was defined as intrapelvic failure (residual tumor regrowth), and distant failure was defined as extrapelvic recurrence. Loco-regional control (LRC) was defined as the time interval from the start of RT for recurrent disease to loco-regional failure. PFS was defined as the time from the start of RT to progression. OS was defined as the time interval from the start of RT to the date of death or last follow-up. Radiation-induced toxicity was reported by using the Common Terminology Criteria for adverse events v.4.03. The symptoms were assessed and recorded at every follow-up visit. Late toxicity was defined as symptoms occurring more than 6 months after salvage RT.

\section{Statistics}

For statistical analyses, IBM SPSS ver. 18.0 (IBM Co., Armonk, NY, USA) was used. Fisher exact tests were used to determine the clinical factors related to treatment failure. Actuarial LRC, PFS, and OS rates were estimated by using the Kaplan-Meier method. Log-rank tests were used to compare clinical variables. The Cox proportional-regression hazard model was used to assess independent prognostic factors for survival. Statistical significance was defined as $\mathrm{P}<0.05$.

\section{RESULTS}

\section{Patient characteristics and treatment options}

Table 1 presents the patients' characteristics. At the time of initial diagnosis of primary cancer, preoperative concurrent chemoradiotherapy was performed in 5 patients and postoperative RT was performed in 3 patients (Table 1). All patients were pathologically diagnosed with an adenocarcinoma, except for 2 patients who had a mucinous carcinoma. The median prior RT dose at initial diagnosis was 50.4 Gy (range, 50.4-54.0 Gy). The median time from initial surgery to the start of salvage RT was 15 months (range, 1.9-50.6 months, with 6 patients having an interval of more than 2 years). There were 15 male patients (68.2\%), and the median age at recurrence was 68 years. The primary disease site was the rectum ( $\mathrm{n}=20,90.9 \%)$ followed by the rectosigmoid colon $(\mathrm{n}=2,9.1 \%)$. All patients who were enrolled in this study had undergone curative surgery of the primary cancer; 17 (77.3\%) had undergone a low anterior resection, 4 had undergone an abdominoperineal resection, and 1 had undergone a segmental re- 
Table 1. Patient characteristics $(n=22)$

\begin{tabular}{|c|c|}
\hline Characteristic & Value \\
\hline \multicolumn{2}{|l|}{ Sex } \\
\hline Male & $15(68.2)$ \\
\hline Female & $7(31.8)$ \\
\hline Age (yr), median (range) & $68(37-82)$ \\
\hline \multicolumn{2}{|l|}{ Initial tumor location } \\
\hline Colon (rectosigmoid) & $2(9.1)$ \\
\hline Rectum & $20(90.9)$ \\
\hline \multicolumn{2}{|l|}{ Initial T stage } \\
\hline pT2 & $2(9.1)$ \\
\hline pT3 & $18(81.8)$ \\
\hline pT4 & $2(9.1)$ \\
\hline \multicolumn{2}{|l|}{ Initial N stage } \\
\hline pNO & $10(45.5)$ \\
\hline $\mathrm{pN}+$ & $12(54.5)$ \\
\hline \multicolumn{2}{|l|}{ Initial pathologic feature } \\
\hline Positive resection margin & $0(0)$ \\
\hline Perinodal extension & $4(18.2)$ \\
\hline Lymphatic invasion & $4(18.2)$ \\
\hline Vascular invasion & $6(27.3)$ \\
\hline Neuronal invasion & $5(22.7)$ \\
\hline \multicolumn{2}{|l|}{ Initial surgery } \\
\hline Low anterior resection & $17(77.3)$ \\
\hline Abdominoperineal resection & $4(18.2)$ \\
\hline Segmental resection & $1(4.5)$ \\
\hline \multicolumn{2}{|c|}{ Interval from initial OP to salvage RT } \\
\hline$<2$ years & $16(72.7)$ \\
\hline$\geq 2$ years & $6(27.3)$ \\
\hline \multicolumn{2}{|l|}{ Recurred site } \\
\hline Axial & $12(54.5)$ \\
\hline Anterior & $2(9.1)$ \\
\hline Lateral & $7(31.8)$ \\
\hline Posterior & $0(0.0)$ \\
\hline Anterior + lateral & $1(4.5)$ \\
\hline \multicolumn{2}{|l|}{ Recurred N stage } \\
\hline rNO & $15(68.2)$ \\
\hline $\mathrm{rN+}$ & 7 (31.8) \\
\hline \multicolumn{2}{|l|}{ Sequence of RT } \\
\hline First & $14(63.6)$ \\
\hline Second (reRT) & $8(36.4)$ \\
\hline \multicolumn{2}{|l|}{ RT dose (Gy) } \\
\hline$<70$ & $13(59.1)$ \\
\hline$\geq 70$ & $9(40.9)$ \\
\hline \multicolumn{2}{|l|}{ Combined CT with RT } \\
\hline Yes & $11(50.0)$ \\
\hline No & $11(50.0)$ \\
\hline
\end{tabular}

Values are presented as number (\%) unless otherwise indicated. OP, operation; RT, radiotherapy; 3D-CRT, 3-dimensional conformal radiotherapy; IMRT, intensity modulated radiotherapy; reRT, re-irradiation; CT, chemotherapy. section with clear resection margins. The most common initial T stage was pT3 $(\mathrm{n}=18,81.8 \%)$. Two patients presented with pT2 stage disease, and 2 other patients presented with pT4 stage at the time of the initial surgery. Twelve patients (54.5\%) showed regional pelvic lymph nodal metastases at the time of the initial pathologic diagnosis. Other pathologic features of patients were reported as follows: 4 with perinodal extension, 4 with lymphatic invasion, 6 with vascular invasion, and 5 with neuronal invasion.

At the time of recurrence, regional nodal recurrence was observed in seven patients; among them, 1 patient showed both local and regional recurrence and the other 6 patients showed only regional (pelvic) nodal recurrence. The remaining 15 patients showed only local recurrence (anastomosis site recurrence with or without invading other structures). The recurrent tumor's location was categorized according to the Guillem classification [12]. An axial location was the most common site of the recurrent tumors $(\mathrm{n}=12,54.5 \%)$ in this study.

Concurrent chemotherapy was administered to 11 patients (50.0\%) along with salvage RT. In addition, 3 patients (13.6\%) received sequential chemotherapy after RT. For salvage RT, the median radiation dose was 57.6 Gy (range, 45-75.6 Gy). Among the 22 patients, 11 patients underwent field reduction at a median dose of $45 \mathrm{~Gy}$ (range, 44-50 Gy) during the treatment. The median total dose was $75.3 \mathrm{~Gy}$ (range, 50.0-118.8 Gy).

Patients were divided into 2 groups according to the type of RT they received: the patients treated with RT for the first time at the time of recurrence without a prior medical history of irradiation (these patients had not receive RT as an initial treatment, $\mathrm{n}=14$ ) versus the patients who were treated with reirradiation $(\mathrm{n}=8)$. For patients without prior irradiation $(\mathrm{n}=14)$, the median radiation dose was $70 \mathrm{~Gy}$ (range, 50-75.6 Gy). For patients who were treated with reirradiation $(\mathrm{n}=8)$, the median radiation dose for salvage RT was $45 \mathrm{~Gy}$ (range, 45-70.2 Gy). Among the 22 patients who were evaluated in this study, 9 patients (40.9\%) received dose-escalated RT ( $\geq 70 \mathrm{~Gy}$ ) for the treatment of recurrent disease in order to improve local control. Among the 9 patients treated with dose-escalated RT, 8 patients received RT for the first time at the moment of recurrence (Fig. 1).

\section{Oncologic outcomes}

The median follow-up was 24.9 months (range, 4.5-66.6 months). After salvage RT, 3 patients showed a complete response and four a partial response. In the imaging studies, 1 patient showed no significant changes (stable disease). Fourteen patients (63.6\%) experienced disease progression (Fig. 1). Six patients died during the follow-up period ( 5 deaths were related to disease progression).

\section{Pattern of failure and prognostic factors}

Treatment failure after salvage RT was observed in 14 patients: 8 had loco-regional failure and 9 had distant metastases (3 patents showed both loco-regional failure and distant failure). The distant 


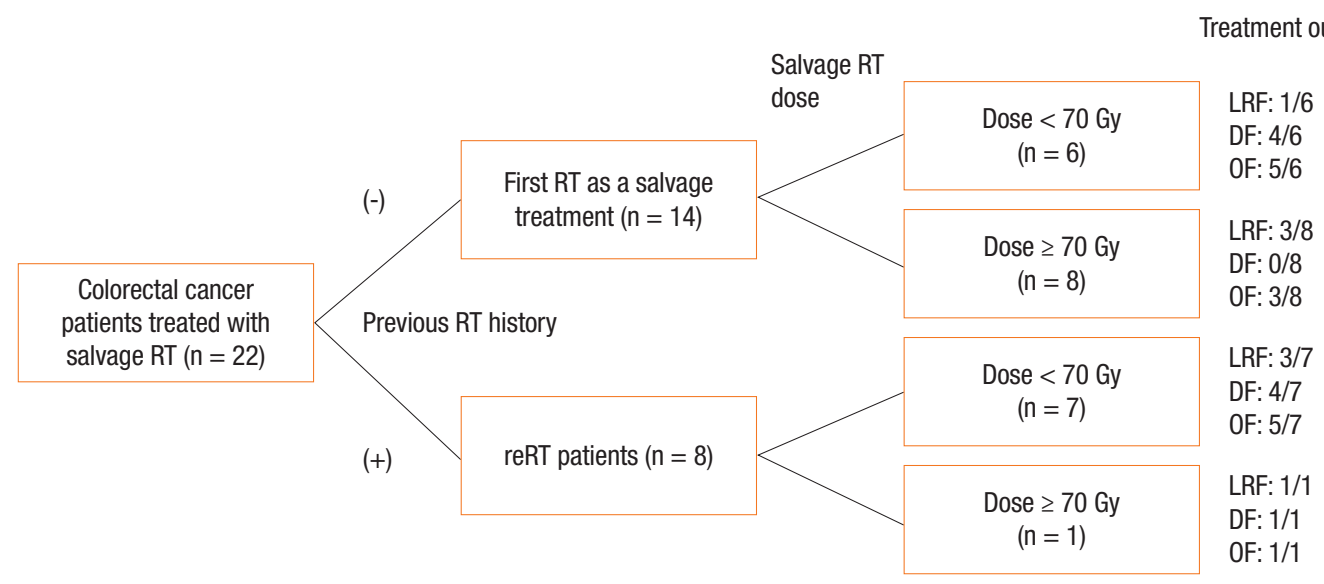

Fig. 1. Patient arrangement and treatment outcomes according to treatment methods. RT, radiotherapy; reRT, reirradiation; LRF, loco-regional failure; DF, distant failure; $\mathrm{OF}$, overall failure.

Table 2. Clinical factors related to treatment failure

\begin{tabular}{|c|c|c|c|c|c|c|}
\hline \multirow{2}{*}{ Variable } & \multicolumn{2}{|c|}{ Loco-regional failure $(n=8)$} & \multicolumn{2}{|c|}{ Distant failure $(\mathrm{n}=9)$} & \multicolumn{2}{|c|}{ Overall failure $(n=14)$} \\
\hline & No. $(\%)$ & P-value & No. $(\%)$ & P-value & No. $(\%)$ & P-value \\
\hline Age (yr) & & 0.183 & & 0.387 & & 1.000 \\
\hline$<68$ & $6 / 11(54.5)$ & & $3 / 11(27.3)$ & & $7 / 11(63.6)$ & \\
\hline$\geq 68$ & 2/11 (18.2) & & $6 / 11(54.5)$ & & $7 / 11(63.6)$ & \\
\hline Recurred site & & 0.675 & & 0.666 & & 1.000 \\
\hline Axial & $5 / 12(41.7)$ & & 4/12 (33.3) & & $8 / 12(66.7)$ & \\
\hline Others & $3 / 10(30.0)$ & & $5 / 10(50.0)$ & & $6 / 10(60.0)$ & \\
\hline Recurred N stage & & 1.000 & & 0.376 & & 1.000 \\
\hline rNO & $5 / 15(33.3)$ & & $5 / 15(33.3)$ & & $9 / 15(60.0)$ & \\
\hline $\mathrm{rN+}$ & $3 / 7(42.9)$ & & $4 / 7(57.1)$ & & $5 / 7(71.4)$ & \\
\hline Sequence of RT & & 0.386 & & 0.187 & & 0.649 \\
\hline First & 4/14 (28.6) & & 4/14 (28.6) & & 8/14 (57.1) & \\
\hline Second (reRT) & $4 / 8(50.0)$ & & $5 / 8(62.5)$ & & $6 / 8(75.0)$ & \\
\hline RT dose (Gy) & & 0.662 & & 0.031 & & 0.187 \\
\hline$<70$ & $4 / 13(30.8)$ & & $8 / 13(61.5)$ & & $10 / 13(76.9)$ & \\
\hline$\geq 70$ & $4 / 9(44.4)$ & & $1 / 9(11.1)$ & & $4 / 9(44.4)$ & \\
\hline Combined CT with RT & & 1.000 & & 0.008 & & 0.183 \\
\hline Yes & 4/11 (36.4) & & 8/11 (72.7) & & $9 / 11(81.8)$ & \\
\hline No & 4/11 (36.4) & & $1 / 11(9.1)$ & & $5 / 11(45.5)$ & \\
\hline Interval from initial OP to salvage RT (yr) & & 0.624 & & 1.000 & & 1.000 \\
\hline$<2$ & $5 / 16(31.3)$ & & $7 / 16(43.8)$ & & $10 / 16(62.5)$ & \\
\hline$\geq 2$ & $3 / 6(50.0)$ & & 2/6 (33.3) & & $4 / 6(66.7)$ & \\
\hline
\end{tabular}

$\mathrm{OP}$, operation; RT, radiotherapy; reRT, reirradiation; CT, chemotherapy.

failure sites were the lungs in 5 patients, the liver in 2 patients, and the brain in 1 patient; peritoneal seeding was observed in 1 patient. Fig. 1 shows patient arrangement and treatment outcomes according to the treatment methods.
Table 2 summarizes the factors affecting treatment failures. No significant factors were associated with loco-regional failure. Distant failure was associated with the salvage RT dose ( $\geq 70$ Gy vs. $<70 \mathrm{~Gy}, \mathrm{P}=0.031$ ) and the use of combined chemotherapy with 


\section{Coloproctology}

\section{Efficacy of Dose-Escalated Radiotherapy for Recurrent Colorectal Cancer}

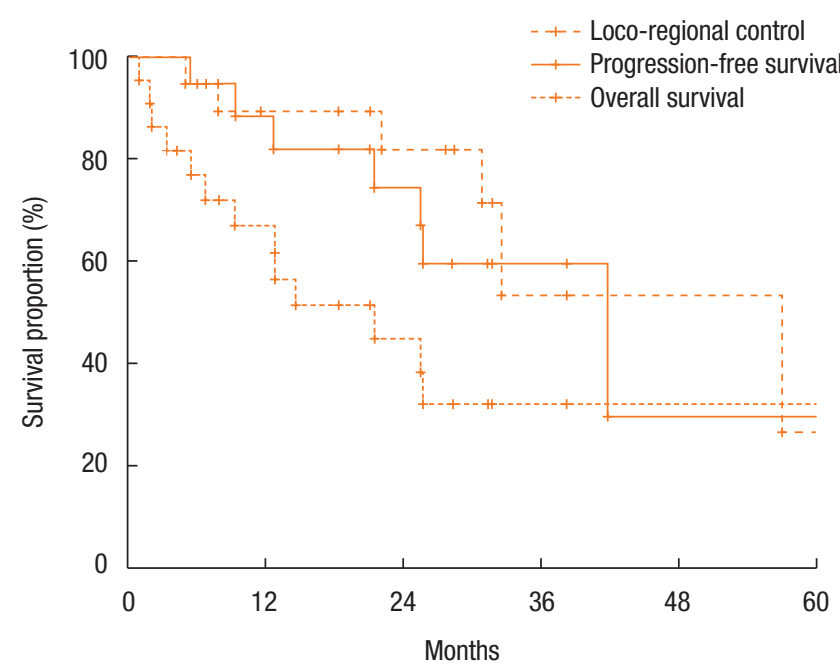

Fig. 2. Loco-regional control, progression-free survival, and overall survival.

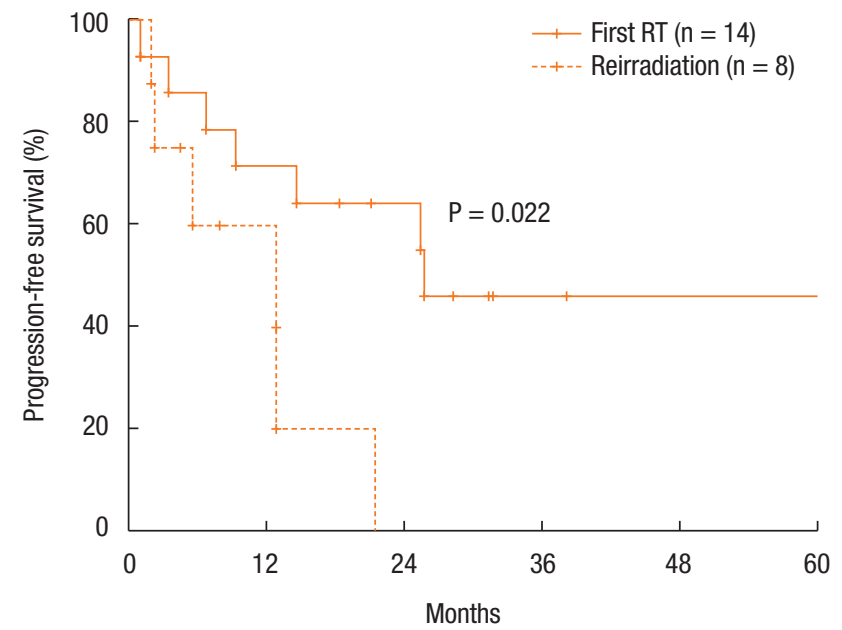

Fig. 3. Progression-free survival according to sequence of radiotherapy (RT) (reirradiation or not).

salvage RT $(\mathrm{P}=0.008)$. Overall progression was not associated with any clinical factors (Table 2).

Fig. 2 shows the actuarial LRC rate of all 22 patients. The 1-year and the 2-year LRC rates were $88.4 \%$ and $74.6 \%$, respectively. Fig. 2 also describes the actuarial PFS and OS rates. The 1-year and the 2-year PFS rates were $67.0 \%$ and $45.1 \%$, respectively (Fig. 2). The 1-year and the 2-year OS rates were $89.4 \%$ and $82.0 \%$, respectively (Fig. 2).

Table 3 summarizes the results of the univariate analyses for the LRC and the PFS. According to the type of RT, the 2-year LRC rate was $79.5 \%$ for patients treated with RT for the first time without prior RT whereas it was $41.7 \%$ for patients treated with reirradiation $(\mathrm{P}=0.101)$ (Table 3). Also, high-dose irradiation $(\geq 70 \mathrm{~Gy})$
Table 3. Prognostic factors in univariate analyses for loco-regional control, progression-free survival, and overall survival

\begin{tabular}{|c|c|c|c|c|}
\hline \multirow[t]{2}{*}{ Factor } & \multirow{2}{*}{ No. } & \multirow{2}{*}{$\begin{array}{c}\text { Loco-regional } \\
\text { control }\end{array}$} & \multirow{2}{*}{$\begin{array}{c}\begin{array}{c}\text { Progression-free } \\
\text { survival }\end{array} \\
\text { P-value }\end{array}$} & \multirow{2}{*}{$\begin{array}{l}\begin{array}{l}\text { Overall } \\
\text { survival }\end{array} \\
\text { P-value }\end{array}$} \\
\hline & & & & \\
\hline Age (yr) & & 0.951 & 0.292 & 0.157 \\
\hline$<68$ & 11 & & & \\
\hline$\geq 68$ & 11 & & & \\
\hline Recurred site & & 0.830 & 0.680 & 0.593 \\
\hline Axial & 12 & & & \\
\hline Others & 10 & & & \\
\hline Recurred N stage & & 0.427 & 0.784 & 0.646 \\
\hline rNO & 14 & & & \\
\hline $\mathrm{rN}+$ & 8 & & & \\
\hline Sequence of RT & & 0.101 & 0.022 & 0.002 \\
\hline First & 14 & & & \\
\hline Second (reRT) & 8 & & & \\
\hline RT dose (Gy) & & 0.640 & 0.014 & 0.270 \\
\hline$<70$ & 13 & & & \\
\hline$\geq 70$ & 9 & & & \\
\hline Combined CT with RT & & 0.979 & 0.132 & 0.574 \\
\hline Yes & 11 & & & \\
\hline No & 11 & & & \\
\hline $\begin{array}{l}\text { Interval from initial OP } \\
\text { to salvage } \mathrm{RT}(\mathrm{yr})\end{array}$ & & 0.185 & 0.960 & 0.137 \\
\hline$<2$ & 16 & & & \\
\hline$\geq 2$ & 6 & & & \\
\hline
\end{tabular}

$\mathrm{RT}$, radiotherapy; reRT, reirradiation; $\mathrm{CT}$, chemotherapy; OP, operation.

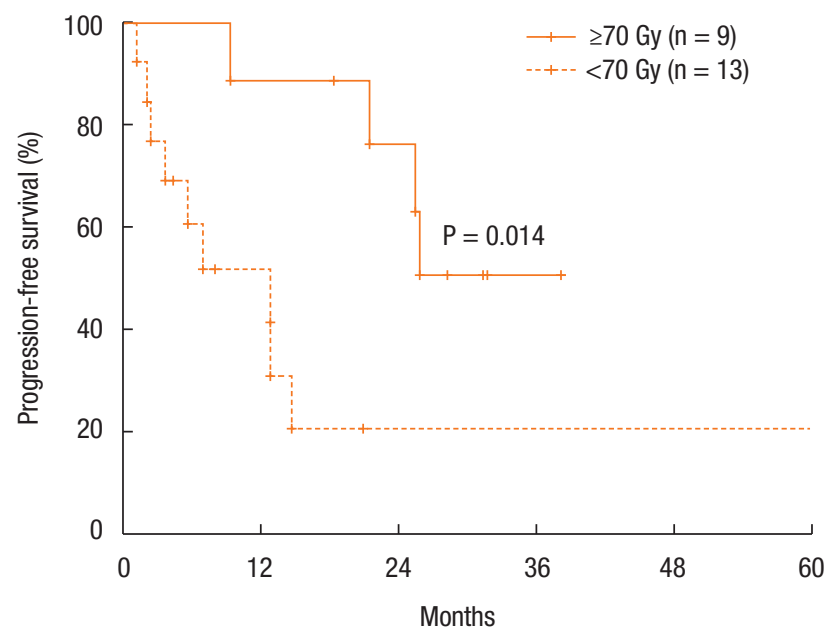

Fig. 4. Progression-free survival according to radiation dose ( $\geq 70$ Gy or $<70$ Gy).

did not result in any significant improvement of the LRC ( $\mathrm{P}=$ 0.640). However, the sequence of RT (reirradiation or not) was as- 
Table 4. Multivariate analysis for progression-free survival

\begin{tabular}{llc}
\hline Variable & \multicolumn{1}{c}{$\mathrm{HR}(95 \% \mathrm{Cl})$} & P-value \\
\hline Sequence of RT, first vs. reRT & $0.273(0.072-1.036)$ & 0.056 \\
RT dose, $\geq 70$ Gy vs. $<70$ Gy & $2.816(0.762-10.405)$ & 0.121 \\
\hline
\end{tabular}

$\mathrm{HR}$, hazard ratio; $\mathrm{Cl}$, confidence interval; $\mathrm{RT}$, radiotherapy; reRT, reirradiation.

sociated with the PFS (Fig. 3). Fig. 3 shows the PFS rate according to the RT sequence (RT for the first time without prior RT vs. reirradiation, $\mathrm{P}=0.022)$. The patients treated with reirradiation showed poor outcomes in the PFS (2-year PFS, RT for the first time without prior RT $64.3 \%$ vs. reirradiation $0 \%, \mathrm{P}=0.022$ ). Fig. 4 shows the benefit of high-dose irradiation ( $\geq 70 \mathrm{~Gy}$ ) for PFS ( $\mathrm{P}=$ 0.014, 2-year PFS $63.5 \%$ vs. $20.8 \%$ ). Reirradiation was also related to poor OS $(\mathrm{P}=0.002)$ (Table 3$)$. However, the multivariate analysis showed no independent prognostic factor for PFS (Table 4).

No grade- 3 or -4 gastrointestinal acute or late toxicities were observed in any of the patients. Also, no specific acute urinary complication was observed in patients during the treatment periods. As for late complications (symptoms occurring 6 months after salvage RT), 4 patients (18.2\%) experienced urinary tract obstruction and underwent surgical intervention. In addition, 1 patient had hematuria. Two patients experienced leg swelling caused by peripheral edema. The total dose ( $>100 \mathrm{~Gy})$ was not associated with radiation-induced late toxicity $(\mathrm{P}=1.000)$.

\section{DISCUSSION}

In this study, we evaluated the clinical outcomes of RT for treating patients with loco-regional recurrent colorectal cancer. Patients receiving salvage RT had better LRC and PFS compared to those receiving only symptom palliation without $\mathrm{RT}$. In particular, the patients who received RT for the first time for treatment of their recurrence showed better PFS and OS than those treated with reirradiation. Therefore, RT can be a possible treatment option for patients who are not suited for curative surgery. In addition, dose escalation ( $\geq 70 \mathrm{~Gy})$ showed a benefit in PFS.

Higher radiation doses ( $\geq 70 \mathrm{~Gy}$ ) are generally required to cure gross tumors. A dose of $70 \mathrm{~Gy}$ is known to control gross tumors in most cancers with epithelial origins; it can also be applied to recurrent colorectal tumors according to the results of this study. However, most dose-volume parameters showed that rectal doses $\geq 60$ Gy are associated with Radiation Therapy Oncology Group grade $\geq 2$ rectal toxicity [13]. In addition, more than $20 \%$ of the rectal volume receiving $>70$ Gy is considered to be highly associated with toxicity [13]. Therefore, radiation-induced toxicity should be monitored in patients who receive dose-escalation.

Recently, Koom et al. [14] showed the efficacy (treatment outcome) and safety (radiation toxicity) of reirradiation; both longer PFS (median, 16 months) and a higher grades 3-4 late toxicity rate (36\%) were observed in reirradiation cases. In addition, they argued that dose escalation might improve LRC in patients who received reirradiation. Although our study showed a worse prognosis in patients with LRC among the patients who were treated with reirradiation, RT for salvage treatment in patients with recurrent colorectal cancer seems to be effective, especially in those who are not able to receive salvage surgery. Moreover, dose escalation seems to be effective for improving clinical outcomes in patients treated with RT.

The reduced rate of distant failure and the improved PFS in patients who received high dose RT ( $\geq 70 \mathrm{~Gy}$ ) may be related to the abscopal effect; local RT inhibits distant, untreated tumors through immunologic mediation [15]. In this regard, further biological studies can be helpful to determine the clear mechanism underlying this phenomenon.

Salvage RT resulted in good LRC and PFS in this study (2-year LRC rate, $74.6 \%$ and PFS rate, $45.1 \%$; Fig. 2), especially in patients without prior irradiation at initial treatment (2-year PFS rate, $64.3 \%)$. Three patients (13.6\%, ranges of radiation dose for recurrent disease 45-74 Gy) who were enrolled in this study survived for more than 3 years after RT for recurrent disease. This result is better than that obtained in a Swedish study by Palmer et al. [8]; in that study, the patients who received only symptom palliation without chemotherapy and/or RT at the time of recurrence showed no survival at the 3-year follow-up. Moreover, Lee et al. [16] previously reported improved clinical outcomes after chemoradiotherapy in patients with locally recurrent rectal cancer (the 5 -year loco-regional relapse-free survival rate was $66.4 \%$, and the OS rate was $48.9 \%)$. Active salvage RT, even without curative resection, seems to be effective and better than supportive care or palliative therapy for this patient population [8].

In contrast to previous reports about the prognostic factors of operable, recurrent colorectal cancer [12], the tumor site (lateral or posterior) was not a determining factor for prognosis in patients treated with RT. In contrast to the results of another study with a multidisciplinary approach [17], the interval of recurrence was not a significant prognostic factor in this study. This may be one of the specific features of RT outcomes. However, drawing conclusions is difficult because the negative results may have been affected by the relatively small sample size of this study.

This study has several limitations. First, the follow-up period was relatively short (median, 24.9 months). Moreover, it was designed as a retrospective study and included a relatively small number of patients $(n=22)$ because of the rarity of the condition. Nevertheless, we showed an improved PFS in patients treated with high-dose radiation. A large-scale, long-term follow-up study should be undertaken to clarify the effect of salvage RT.

For future studies of RT for the treatment of patients with colorectal cancer recurrence, preoperative RT combined with reoperation [18] might be an interesting topic. Symptom evaluation may also be an important issue, although we could not deal with the effect of pain palliation caused by salvage RT in this study owing to the lack of access to medical records. Dose escalation using an IMRT technique also seems to be a promising avenue of re- 
search [19]. Attempts at dose escalation using IMRT to improve local control and reduce the rate of radiation toxicity should be continued. In addition, the use of stereotactic body RT for the treatment of patients with oligo-recurrence in the pelvis may be another treatment option [20], and it should be evaluated further.

In conclusion, salvage RT for the treatment of patients with locally-recurrent colorectal cancer can be offered when surgery is not possible, especially in patients who did not receive prior RT as an initial treatment. Furthermore, dose escalation seems to have a potential benefit for the treatment outcome. Further evaluation is required to assess the effect of RT for the treatment of patients with recurrent colorectal cancer.

\section{CONFLICT OF INTEREST}

No potential conflict of interest relevant to this article was reported.

\section{REFERENCES}

1. Benson AB 3rd, Bekaii-Saab T, Chan E, Chen YJ, Choti MA, Cooper HS, et al. Rectal cancer. J Natl Compr Canc Netw 2012;10: 1528-64.

2. Peeters KC, Marijnen CA, Nagtegaal ID, Kranenbarg EK, Putter $\mathrm{H}$, Wiggers $\mathrm{T}$, et al. The TME trial after a median follow-up of 6 years: increased local control but no survival benefit in irradiated patients with resectable rectal carcinoma. Ann Surg 2007;246: 693-701.

3. Pilipshen SJ, Heilweil M, Quan SH, Sternberg SS, Enker WE. Patterns of pelvic recurrence following definitive resections of rectal cancer. Cancer 1984;53:1354-62.

4. Wolmark N, Fisher B. An analysis of survival and treatment failure following abdominoperineal and sphincter-saving resection in Dukes' B and C rectal carcinoma. A report of the NSABP clinical trials. National Surgical Adjuvant Breast and Bowel Project. Ann Surg 1986;204:480-9.

5. Moriya Y. Treatment strategy for locally recurrent rectal cancer. Jpn J Clin Oncol 2006;36:127-31.

6. Mohiuddin M, Marks GM, Lingareddy V, Marks J. Curative surgical resection following reirradiation for recurrent rectal cancer. Int J Radiat Oncol Biol Phys 1997;39:643-9.

7. Bouchard P, Efron J. Management of recurrent rectal cancer. Ann Surg Oncol 2010;17:1343-56.
8. Palmer G, Martling A, Cedermark B, Holm T. A populationbased study on the management and outcome in patients with locally recurrent rectal cancer. Ann Surg Oncol 2007;14:447-54.

9. Wong CS, Cummings BJ, Brierley JD, Catton CN, McLean M, Catton $\mathrm{P}$, et al. Treatment of locally recurrent rectal carcinoma-results and prognostic factors. Int J Radiat Oncol Biol Phys 1998; 40:427-35.

10. Nielsen MB, Laurberg S, Holm T. Current management of locally recurrent rectal cancer. Colorectal Dis 2011;13:732-42.

11. Tanis PJ, Doeksen A, van Lanschot JJ. Intentionally curative treatment of locally recurrent rectal cancer: a systematic review. Can J Surg 2013;56:135-44.

12. Moore HG, Shoup M, Riedel E, Minsky BD, Alektiar KM, Ercolani $\mathrm{M}$, et al. Colorectal cancer pelvic recurrences: determinants of resectability. Dis Colon Rectum 2004;47:1599-606.

13. Michalski JM, Gay H, Jackson A, Tucker SL, Deasy JO. Radiation dose-volume effects in radiation-induced rectal injury. Int J Radiat Oncol Biol Phys 2010;76(3 Suppl):S123-9.

14. Koom WS, Choi Y, Shim SJ, Cha J, Seong J, Kim NK, et al. Reirradiation to the pelvis for recurrent rectal cancer. J Surg Oncol 2012; 105:637-42.

15. Demaria S, Ng B, Devitt ML, Babb JS, Kawashima N, Liebes L, et al. Ionizing radiation inhibition of distant untreated tumors (abscopal effect) is immune mediated. Int J Radiat Oncol Biol Phys 2004;58:862-70.

16. Lee JH, Kim DY, Kim SY, Park JW, Choi HS, Oh JH, et al. Clinical outcomes of chemoradiotherapy for locally recurrent rectal cancer. Radiat Oncol 2011;6:51.

17. Zhao J, Du CZ, Sun YS, Gu J. Patterns and prognosis of locally recurrent rectal cancer following multidisciplinary treatment. World J Gastroenterol 2012;18:7015-20.

18. Valentini V, Morganti AG, Gambacorta MA, Mohiuddin M, Doglietto GB, Coco C, et al. Preoperative hyperfractionated chemoradiation for locally recurrent rectal cancer in patients previously irradiated to the pelvis: A multicentric phase II study. Int J Radiat Oncol Biol Phys 2006;64:1129-39.

19. Cai G, Zhu J, Hu W, Zhang Z. Accelerated hyperfractionated intensity-modulated radiotherapy for recurrent/unresectable rectal cancer in patients with previous pelvic irradiation: results of a phase II study. Radiat Oncol 2014;9:278.

20. Seo YS, Kim MS, Yoo HJ, Jang WI. Stereotactic body radiotherapy for oligo-recurrence within the nodal area from colorectal cancer. World J Gastroenterol 2014;20:2005-13. 